Bull. Korean Math. Soc. 51 (2014), No. 2, pp. 547-553

http://dx.doi.org/10.4134/BKMS.2014.51.2.547

\title{
ON ZEROS OF THE BOUBAKER POLYNOMIALS
}

\author{
SEON-HONG KIM
}

Abstract. The Boubaker polynomials arose from the discretization of the equations of heat transfer in pyrolysis starting from an assumed solution of the form

$$
\frac{1}{N} e^{\frac{A}{H / z+1}} \sum_{k=0}^{\infty} \xi_{k} J_{k}(t),
$$

where $J_{k}$ is the $k$-th order Bessel function of the first kind. In this paper, we investigate the distribution of zeros of the Boubaker polynomials.

\section{Introduction}

The Boubaker polynomials are defined by

$$
B_{n}(x)=\sum_{j=0}^{\lfloor n / 2\rfloor}(-1)^{j} \frac{n-4 j}{n-j}\left(\begin{array}{c}
n-j \\
j
\end{array}\right) x^{n-2 j}
$$

and satisfy the recurrence relations

$$
\begin{aligned}
& B_{0}(x)=1, \quad B_{1}(x)=x, \quad B_{2}(x)=x^{2}+2, \\
& B_{n}(x)=x B_{n-1}(x)-B_{n-2}(x), \quad n \geq 3 .
\end{aligned}
$$

The first few terms of this polynomial sequence starting from $B_{3}(x)$ are

$$
\begin{aligned}
& B_{3}(x)=x^{3}+x, \\
& B_{4}(x)=x^{4}-2, \\
& B_{5}(x)=x^{5}-x^{3}-3 x, \\
& B_{6}(x)=x^{6}-2 x^{4}-3 x^{2}+2, \\
& B_{7}(x)=x^{7}-3 x^{5}-2 x^{3}+5 x, \\
& B_{8}(x)=x^{8}-4 x^{6}+8 x^{2}-2 .
\end{aligned}
$$

Received January 5, 2013; Revised June 27, 2013

2010 Mathematics Subject Classification. Primary 30C15; Secondary 26C10.

Key words and phrases. Boubaker polynomials, zeros.

This research was supported by the Sookmyung Women's University Research Grants 2012 . 
The Boubaker polynomials arose from the discretization of the equations of heat transfer in pyrolysis ([2], [5], [6]) starting from an assumed solution of the form

$$
\frac{1}{N} e^{\frac{A}{H / z+1}} \sum_{k=0}^{\infty} \xi_{k} J_{k}(t),
$$

where $J_{k}$ is the $k$-th order Bessel function of the first kind, and the Boubaker Polynomials Expansion Scheme BPES has been used by several applied physics and engineering studies. For references, see [1], [3], [4], [5], [6], [7], [9], [10].

In this paper, we study the Boubaker polynomials from a rather mathematical point of view. Perhaps one of the fundamental questions about polynomials is to investigate how the zeros of polynomials are located in the complex plane. It is the purpose of the present paper to investigate the distribution of zeros of the Boubaker polynomials.

There have been similar results that are Theorem 3.1, Theorem 5.1, Remark 5.1 , Corollary 5.1 in [12] to some of ours. But we have some doubts in the proof of Theorem 5.1. The proof is based upon

$$
B_{n}(2 \cos t)=4 \cos t \sin (n t)-2 \cos (n t)
$$

and

$$
\tan t=2 \tan (n t)
$$

But a numerical example shows that (2) is not true. Also for (3) being true, it seems to be

$$
B_{n}(2 \cos t)=4 \cos t \sin (n t)-2 \cos (n t) \sin t
$$

instead of (2) but this is not true either. Questioning of the proof of Theorem 5.1 does not allow us to believe in Remark 5.1. Also it is hard to understand how the authors know that, in Remark 5.1 and Theorem 5.2, the two nonreal complex zeros are purely imaginary. They stated these in Remark 5.1 without proofs. Finally there seems to be a minor error in Theorem 3.1. Following their proofs of Theorem 3.1, we can see that the result is $\left|x_{i}\right| \leq 2$ instead of $\left|x_{i}\right|<2$. Our all corresponding proofs will be totally different from those in [12].

Before studying our main topics, we first remark their resultants. The resultant of two polynomials is in general a rather complicated function of their coefficients. However, there is an elegant formula for the resultant of any two consecutive Boubaker polynomials due to I. Schur (see $\S 6.71$ of [11]). In fact, using Schur's result we can check that for $n \geq 2$, the resultant of $B_{n}(x)$ and $B_{n-1}(x)$ is

$$
(-1)^{\left(\begin{array}{l}
n \\
2
\end{array}\right)-1} \cdot 2 \text {. }
$$

This means that if $x_{j, n}(1 \leq j \leq n)$ are the zeros of $B_{n}(x)$, then

$$
\prod_{j=1}^{n} B_{n-1}\left(x_{j, n}\right)=(-1)^{\left(\begin{array}{l}
n \\
2
\end{array}\right)-1} \cdot 2 \text {. }
$$




\section{Results and proofs}

We begin with obtaining a new representation of Boubaker polynomials. This will be useful in the proof of Theorem 2.4.

Proposition 2.1. We have

$$
\begin{aligned}
B_{n}(x)= & -\frac{1}{\sqrt{x^{2}-4}}\left[\frac { 1 } { 2 ^ { n } } \left(2 x\left(\left(x-\sqrt{x^{2}-4}\right)^{n}-\left(x+\sqrt{x^{2}-4}\right)^{n}\right)\right.\right. \\
& \left.\left.+\sqrt{x^{2}-4}\left(\left(x-\sqrt{x^{2}-4}\right)^{n}+\left(x+\sqrt{x^{2}-4}\right)^{n}\right)\right)\right] .
\end{aligned}
$$

Proof. By (1), we see

$$
\left(\begin{array}{c}
B_{n}(x) \\
B_{n-1}(x)
\end{array}\right)=\left(\begin{array}{cc}
x & -1 \\
1 & 0
\end{array}\right)\left(\begin{array}{l}
B_{n-1}(x) \\
B_{n-2}(x)
\end{array}\right)
$$

and

$$
\left(\begin{array}{c}
B_{n}(x) \\
B_{n-1}(x)
\end{array}\right)=\left(\begin{array}{cc}
x & -1 \\
1 & 0
\end{array}\right)^{n-2}\left(\begin{array}{l}
B_{2}(x) \\
B_{1}(x)
\end{array}\right)=\left(\begin{array}{cc}
x & -1 \\
1 & 0
\end{array}\right)^{n-2}\left(\begin{array}{c}
x^{2}+2 \\
x
\end{array}\right)
$$

Let

$$
A=\left(\begin{array}{cc}
x & -1 \\
1 & 0
\end{array}\right)
$$

Then the eigenvalues of matrix $A$ are

$$
\lambda_{1}=\frac{x-\sqrt{x^{2}-4}}{2}, \quad \lambda_{2}=\frac{x+\sqrt{x^{2}-4}}{2},
$$

and hence

This implies

$$
\left(\begin{array}{cc}
x & -1 \\
1 & 0
\end{array}\right)=\left(\begin{array}{cc}
\lambda_{1} & \lambda_{2} \\
1 & 1
\end{array}\right)\left(\begin{array}{cc}
\lambda_{1} & 0 \\
0 & \lambda_{2}
\end{array}\right)\left(\begin{array}{cc}
\lambda_{1} & \lambda_{2} \\
1 & 1
\end{array}\right)^{-1}
$$

$$
\left(\begin{array}{cc}
x & -1 \\
1 & 0
\end{array}\right)^{n-2}=\frac{1}{\lambda_{1}-\lambda_{2}}\left(\begin{array}{cc}
\lambda_{1} & \lambda_{2} \\
1 & 1
\end{array}\right)\left(\begin{array}{cc}
\lambda_{1}^{n-2} & 0 \\
0 & \lambda_{2}^{n-2}
\end{array}\right)\left(\begin{array}{cc}
1 & -\lambda_{2} \\
-1 & \lambda_{1}
\end{array}\right) .
$$

Substituting this into (4) yields

$$
\left(\begin{array}{c}
B_{n}(x) \\
B_{n-1}(x)
\end{array}\right)=\frac{1}{\lambda_{1}-\lambda_{2}}\left(\begin{array}{cc}
\lambda_{1}^{n-1}-\lambda_{2}^{n-1} & -\lambda_{1}^{n-1} \lambda_{2}+\lambda_{1} \lambda_{2}^{n-1} \\
\lambda_{1}^{n-2}-\lambda_{2}^{n-2} & -\lambda_{1}^{n-2} \lambda_{2}+\lambda_{1} \lambda_{2}^{n-2}
\end{array}\right)\left(\begin{array}{c}
x^{2}+2 \\
x
\end{array}\right),
$$

and by direct computation using (5), the result follows.

Remarks. In the proof of above proposition, we obtained

$$
B_{n}(x)=\frac{1}{\lambda_{1}-\lambda_{2}}\left(2\left(\lambda_{1}+\lambda_{2}\right)\left(\lambda_{1}^{n}-\lambda_{2}^{n}\right)-\left(\lambda_{1}-\lambda_{2}\right)\left(\lambda_{1}^{n}+\lambda_{2}^{n}\right)\right) .
$$

By simple computations, we may see that the zeros $x$ of $B_{n}(x)$ satisfy the homogeneous equation in $\lambda_{1}$ and $\lambda_{2}$

$$
\lambda_{1}^{n+1}-\lambda_{2}^{n+1}-3 \lambda_{1} \lambda_{2}^{n}+3 \lambda_{1}^{n} \lambda_{2}=0,
$$


and

$$
\left(\frac{\lambda_{1}}{\lambda_{2}}\right)^{n+1}-1-3\left(\frac{\lambda_{1}}{\lambda_{2}}\right)+3\left(\frac{\lambda_{1}}{\lambda_{2}}\right)^{n}=0 .
$$

With

$$
z:=\frac{\lambda_{1}}{\lambda_{2}}=\frac{x-\sqrt{x^{2}-4}}{x+\sqrt{x^{2}-4}},
$$

the above equation can be rewritten by

$$
z^{n+1}+3 z^{n}-3 z-1=0 .
$$

Also from (6) we can get a relation between $x$ and $z$

$$
x^{2}=2+z+\frac{1}{z} \text {. }
$$

From (7), we let

$$
p_{n}(z)=z^{n+1}+3 z^{n}-3 z-1
$$

and investigate their zero distribution to obtain zero distribution results of $B_{n}(x)$. For this we need the following theorem about self-inversive polynomials. A polynomial $P_{n}(z)=a_{n} z^{n}+a_{n-1} z^{n-1}+\cdots+a_{0}$ with real coefficients is said to be a self-inversive polynomial of degree $n$ if it satisfies $a_{n} \neq 0$ and $P_{n}(z)= \pm z^{n} P_{n}(1 / z)$. The polynomial $p_{n}(z)$ above is self-inversive.

Theorem 2.2 (Cohn). Let $f(z)$ be a self-inversive polynomial of degree $n$. Suppose that $f(z)$ has exactly $\tau$ zeros on the unit circle (counted according to multiplicity) and exactly $\nu$ critical points in the closed unit disc (counted according to multiplicity). Then

$$
\tau=2(\nu+1)-n
$$

We may see above theorem in p. 230 of [8]. Using this theorem, we obtain the following. Throughout the rest, $U$ denotes the unit circle for convenience and $n$ is a positive integer greater than 5 .

Proposition 2.3. If $n$ is odd, $p_{n}(z)$ has four real zeros

$$
1,-1, z_{0}, \frac{1}{z_{0}} \quad\left(-3<z_{0}<-3+\frac{1}{n}\right)
$$

and all the others are nonreal complex zeros on $U$. If $n$ is even, $p_{n}(z)$ has three real zeros

$$
1, z_{1}, \frac{1}{z_{1}} \quad\left(-3-\frac{1}{n}<z_{1}<-3\right)
$$

and all the others are nonreal complex zeros on $U$. 
Proof. We observe that

$$
p_{n}(z)=(z-1)\left(z^{n}+4 z^{n-1}+4 z^{n-2}+\cdots+4 z+1\right)
$$

is a self-inversive polynomial. By Descartes' rule of signs, $p_{n}(z)$ has only one positive real zero 1 . Consider

$$
p_{n}^{\prime}(z)=(n+1) z^{n}+3 n z^{n-1}-3 .
$$

Rouché's theorem guarantees that $p_{n}^{\prime}(z)$ has $n-1$ zeros in the open unit disk $|z|<1$ since for $z \in U$,

$$
3 n|z|^{n-1}=3 n>(n+1)+3 \geq\left|(n+1) z^{n}-3\right| .
$$

Then by Theorem 2.2, $p_{n}(z)$ has $n-1$ zeros on $U$. If $n$ is odd, it is easy to check that

$$
p_{n}(0)<0, p_{n}( \pm 1)=0, p_{n}^{\prime}(-1)=-(n+1)+3 n-3=2(n-2)>0,
$$

and for $x>1$ we have $p_{n}(x)>0$ and $p_{n}(x) \rightarrow+\infty$ as $x \rightarrow-\infty$. So there are at least two negative real zeros $z_{0}$ and $z_{0}^{\prime}$ other than -1 where

$$
z_{0}<-1, \quad-1<z_{0}^{\prime}
$$

and $z_{0}^{\prime}=1 / z_{0}$ because $p_{n}(z)$ is self-inversive. Since the polynomial $p_{n}(z)$ of degree $n+1$ has $n-1$ zeros on $U$ and $p_{n}( \pm 1)=0, p_{n}(z)$ has four real zeros $1,-1, z_{0}, \frac{1}{z_{0}}$ and all the others are nonreal complex zeros on $U$. Similarly, we can show results for the case $n$ even. It remains to obtain the bounds for real zeros other than \pm 1 . We first note that $p_{n}(-3)=8>0$. Suppose $n$ is odd. Using a well-known inequality: for $x>-1,(1+x)^{n} \geq 1+n x$,

$$
\begin{aligned}
p_{n}\left(-3+\frac{1}{n}\right) & =\left(-3+\frac{1}{n}\right)^{n+1}+3\left(-3+\frac{1}{n}\right)^{n}-3\left(-3+\frac{1}{n}\right)-1 \\
& =\left(3-\frac{1}{n}\right)^{n}\left(3-\frac{1}{n}-3\right)+3\left(3-\frac{1}{n}\right)-1 \\
& =-\frac{1}{n}\left(3-\frac{1}{n}\right)^{n}+8-\frac{3}{n}=-\frac{3^{n}}{n}\left(1-\frac{1}{3 n}\right)^{n}+8-\frac{3}{n} \\
& \leq-\frac{3^{n}}{n}\left(1-\frac{n}{3 n}\right)+8-\frac{3}{n}=-\frac{2 \cdot 3^{n-1}}{n}+8-\frac{3}{n} \\
& <0 .
\end{aligned}
$$

This implies that

For $n$ even,

$$
-3<z_{0}<-3+\frac{1}{n}
$$

$$
\begin{aligned}
p\left(-3-\frac{1}{n}\right) & =\left(-3-\frac{1}{n}\right)^{n+1}+3\left(-3-\frac{1}{n}\right)^{n}-3\left(-3-\frac{1}{n}\right)-1 \\
& =\left(3+\frac{1}{n}\right)^{n}\left(-3-\frac{1}{n}+3\right)+8+\frac{3}{n}
\end{aligned}
$$




$$
\begin{aligned}
& =-\frac{1}{n}\left(3+\frac{1}{n}\right)^{n}+8+\frac{3}{n}=-\frac{3^{n}}{n}\left(1+\frac{1}{3 n}\right)^{n}+8+\frac{3}{n} \\
& \leq-\frac{3^{n}}{n} \cdot \frac{1}{3}+8+\frac{3}{n}=-\frac{3^{n-1}}{n}+8+\frac{3}{n} \\
& <0,
\end{aligned}
$$

which implies that

$$
-3-\frac{1}{n}<z_{1}<-3 .
$$

Now we prove the main result of the paper.

Theorem 2.4. The polynomial $B_{n}(x)$ has $n-2$ real zeros and two nonreal complex zeros that are purely imaginary. All zeros of $B_{n}(x)$ lie in $|x| \leq 2$, and the two purely imaginary zeros lie outside $U$. More specifically, the two purely imaginary zeros, say $i b_{0}$ and $-i b_{0}$, satisfy

$$
\begin{cases}\sqrt{\frac{4 n-3}{3 n}}<b_{0}<\sqrt{\frac{4 n-1}{3 n-1}}, & \text { if } n \text { is odd, } \\ \sqrt{\frac{4 n+1}{3 n+1}}<b_{0}<\sqrt{\frac{4 n+3}{3 n}}, & \text { if } n \text { is even. }\end{cases}
$$

Proof. We recall that by (8),

$$
x^{2}=2+z+\frac{1}{z}
$$

where $x$ is a zero of $B_{n}$ and $z$ is a zero of $p_{n}$. By Proposition 2.3, all zeros of $p_{n}(z)$ on $U$ except real zeros are nonreal complex numbers. For such zeros $z$,

$$
|x|^{2}=\left|2+z+\frac{1}{z}\right| \leq 4
$$

and so $|x| \leq 2$. Also when $z= \pm 1,|x| \leq 2$. For $n$ odd and $-3<z_{0}<-3+\frac{1}{n}$, we can compute that

$$
-\frac{4 n-1}{3 n-1}<x_{0}^{2}=2+z_{0}+\frac{1}{z_{0}}<-\frac{4 n-3}{3 n}
$$

and so $x_{0}$ is a purely imaginary zero of $B_{n}$ and with $x_{0}=i b_{0}$,

$$
1<\sqrt{\frac{4 n-3}{3 n}}<\left|b_{0}\right|<\sqrt{\frac{4 n-1}{3 n-1}} .
$$

Since $p_{n}(z)$ is a polynomial with real coefficients, one more purely imaginary zero of $B_{n}$ must be $-i b_{0}$. The case $n$ even can be proved in the same way.

Example 2.5. The two nonreal purely imaginary zeros $\pm i b_{0}= \pm i 1.15328 \ldots$ of $B_{7}(x)$ satisfy

$$
1.0910 \cdots<b_{0}<1.1619 \cdots .
$$

The two nonreal purely imaginary zeros $\pm i b_{1}= \pm i 1.15517 \cdots$ of $B_{8}(x)$ satisfy

$$
1.1489 \cdots<b_{1}<1.2076 \cdots \text {. }
$$




\section{References}

[1] O. B. Awojoyogbe and K. Boubaker, A solution to Bloch NMR flow equations for the analysis of hemodynamic functions of blood flow system using $m$-Boubaker polynomials, Current Appl. Phys. 9 (2009), no. 1, 278-283.

[2] K. Boubaker, A. Chaouachi, M. Amlouk, and H. Bouzouita, Enhancement of pyrolysis spray dispersal performance using thermal time-response to precursor uniform deposition, Eur. Phys. J. Appl. Phys. 37 (2007), 105-109.

[3] J. Ghanouchi, H. Labiadh, and K. Boubaker, An attempt to solve the heat transfer equation in a model of pyrolysis spray using $4 q$-order $m$-Boubaker polynomials, Int. J. Heat Tech. 26 (2008), 49-52.

[4] T. Ghrib, K. Boubaker, and M. Bouhafs, Investigation of thermal diffusivity-microhardness correlation extended to surface-nitrured steel using Boubaker polynomials expansion, Mod. Phys. Lett. B 22 (2008), no. 29, 2893-2907.

[5] H. Labiadh and K. M. Boubaker, A Sturm-Liouville shaped characteristic differential equation as a guide to establish a quasi-polynomial expression to the Boubaker polynomials, Differ. Uravn. Protsessy Upr. 2007 (2007), no. 2, 117-133.

[6] H. Labiadh, M. Dada, O. B. Awojoyogbe, K. B. Ben Mahmoud, and A. Bannour, Establishment of an ordinary generating function and a Christoffel-Darboux type first order differential equation for the heat equation related Boubaker-Turki polynomials, Differ. Uravn. Protsessy Upr. 2008 (2008), no. 1, 51-66.

[7] S. Lazzez and K. B. Ben Mahmoud, New ternary compounds stoichiometry-linked thermal behavior optimization using Boubaker polynomials, J. Alloys Compounds 476 (2009), 769-773.

[8] T. Sheil-Small, Complex Polynomials, Cambridge Studies in Advanced Mathematics 73, Cambridge University Press, Cambridge, 2002.

[9] S. Slama, J. Bessrour, K. Boubaker, and M. Bouhafs, A dynamical model for investigation of A3 point maximal spatial evolution during resistance spot welding using Boubaker polynomials, Eur. Phys. J. Appl. Phys. 44 (2008), 317-322.

[10] S. Slama, M. Bouhafs, and K. B. Ben Mahmoud, A Boubaker polynomials solution to heat equation for monitoring $A_{3}$ point evolution during resistance spot welding, Int. J. Heat Tech. 26 (2008), 141-146.

[11] G. Szegö, Orthogonal Polynomials, (fourth ed.). Providence (RI): Amer. Math. Soc. 1975.

[12] T. Zhao, B. K. Ben Mahmoud, M. A. Toumi, O. P. Faromika, M. Dada, O. B. Awojoyogbe, J. Magnuson, and F. Lin, Some new properties of the applied-physics related Boubaker polynomials, Differ. Uravn. Protsessy Upr. (2009), no 1, 7-19.

Department of Mathematics

SOOKMYUNG WOMEN'S UNIVERSITY

SEOUL 140-742, KoreA

E-mail address: shkim17@sookmyung.ac.kr 